\title{
LA DIFFUSION ET LA PRODUCTION DE LA CANNE À SUCRE (XIII'-XVI' SIÈCLES)
}

\author{
JaCQUeline GuIRAL HaDZIIOSSIF \\ Université de Paris-Nanterre
}

(France)

Avant d'aborder l'étude des origines et du développement de l'industrie sucrière à Valence à la fin du Moyen Age, je voudrais rappeler quelques problemes historiographiques concernant les modes de production de la canne à sucre au Moyen Age, la diffusion de cette culture et l'origine des innovations technologiques dans ce domaine.

Dans le monde musulman la canne, d'abord plante de jardin cultivée sur une faible échelle, devient l'objet d'une culture de plantation, non plus sur les terres des ortolans mais dans le cadre des domaines de l'état ou des grands. Les opinions divergent sur les modes de travail utilisés pour produire la canne. Selon Claude Cahen ${ }^{1}$, les plantations de cannes se réalisent à travers le travail des paysans libres et les formes de métayage en usage alors, avec un partage variable des récoltes selon l'apport respectif dos contractants.Dans le cas des contrats impliquant l'irrigation comme pour la canne à sucre, le produit peut être partagé par moitié (musaqât), en cas de contrat de complant, la plantation lorsqu'elle rend est divisée selon un pourcentage fixé à l'avance (mugharasa). Dans cette perspective, ce sont les paysans libres eux-mêmes qui réalisent la culture des cannes sur les domaines des grands ou de l'état, il n'y aurait pas eu d'esclavage agricole

'C. CAHEN, L'Islain des origines au début de l'Empire ottoman, Paris, ed. Bordas 1970. pp. 106-107 et pp. 110-113.

"Anuario de Eatudios Medievales", 24 (1994) 
dans les plantations. Il accepte une seule exception cependant au milieu du IX $^{e}$ siècle, la constitution de plantations de cannes sur le bas Iraq, sur les domaines des grands qui importent des esclaves noirs de Zanzibar et d'Afrique orientale pour accomplir les différentes tâches. La révolte de ces esclaves aboutit à une véritable guerre de 869 à 883, qui se termina par leur extermination.

A l'opposé, Noël Deerr estime fort improbable que le sucre produit par les Latins au Moyen Age dans le sud de l'Europe, en Méditerranée orientale à partir du XIII ${ }^{e}$ siècle, n'ait pas été le résultat d'un mode de production esclavagiste. Pourtant il demeure prudent, car il constate qu'on ne dispose d'aucune source permettant d'étayer cette hyputhèse ${ }^{2}$. Moins prudent, dans un livre récent Sydney W. Mintz accepte comme un fait établi l'existence du travail servile dans les domaines sucriers, il attribue à l'arrivée des croisés latins l'intensification de l'esclavage sur les plantations sucriéres de la Méditerranée. Le passage d'une hypothèse à une quasi certitude aboutit ainsi à l'élaboration de l'idée selon laquelle le travail servile était courant dans le domaine musulman, que ce mode de production aurait été transmis aux Latins, que le lien entre le cycle de la canne à sucre et l'esclavage aurait été forgé en Crète et a Chypre. C'est ce modèle préexistant qui aurait été transporté par les occidentaux vers les îles de l'Afrique atlantique ${ }^{3}$. On peut se demander si ces exemples, qui auraient été un exception dans le monde islamique, n'ont pas influencé la vision des historiens, eux-mêmes témoins des expériences coloniales du sucre atlantique débutant à la fin du $X V^{e}$ siècle. Un second problème historiographiques est celui de la diffusion de la canne et de son aire d'implantation. La canne connue dans le golfe persique à l'époque de la conquête arabe se serait répandue dans les zones de plaines chaudes et irriguées. Mais à ces conditions générales s’ajoute la réalisation d'un choix délibéré d'introduire une culture dans des zones où il existe déjà un savoir faire horticole, un réseau d'irrigation ou la possibilité de le réaliser, la présence d'une paysannerie capable d'assurer le cycle de culture de la canne échelonné sur trois années, la volonté de seigneurs et de propriétaires fonciers de substituer à des cultures vivrières ou spéculatives existantes une

${ }^{2}$ N. DIEFR, The History of Sugar, 2 vol. Londres 1950, vol. 2, p. 288.

${ }^{3}$ S.W. MINTZ, Sucre blanc, misère noire, le goût et le pouvoir, Paris, Nathan, 1991, p. 27 et J. H. GALLWAY, The Mediterranean Sugar Industry, "The Geographical Revicw", 67 (1977), pp. 186-190 adopte un point de vue similaire. 
culture spéculative nouvelle, qui puisse être assurée de trouver un débouché immédiat dans les grands courants commerciaux de l'époque. Cette trame n'a malheureusement laissé que peu de traces. Les lacunes des sources écrites ne doivent pas masquer les lacunes de l'historiographie sur le probleme de la diffusion de cette plante dans les îles de la Méditerranée orientale. Dans sa thèse consacrée a la Romanie vénitienne au Moyen $\mathrm{Age}^{4}$, Freddy Thiriet ne fait allusion a la culture de la canne en Crète qu'à deux reprises. Une première mention en 1334 fait état du transport à Venise du "sucre fait et réalisé dans notre île de Crète". la seconde mention est une licence concédée à Marco do Zanono pour planter des cannes et pour construire deux moulins en Crète entre 1428-1431. Or, il affirme que la culture de la canne avait été très médiocre jusque là et qu'il fallait voir dans cette concession les efforts de certains citoyens vénéto-candiotes. La lacune dans la documentation de l'auteur ne suppose pas qu'il y ait eu aussi une lacune dans la culture des cannes a sucre. On aboutit ainsi à des "factoïdes" qui se basent sur l'enchaînement d'affirmations des auteurs successifs, comme le releve très justement Marie Louise von Wartburg. A juste titre cette dernière souligne combien les sources écrites traditionnelles donnnent une image étroite de cette production, image corrigée grâce à ce premier projet de recherche en archéologie industrielle médiévale a Chypres. Le dernier probleme historiographique est lié a la technologie employée dans les raffineries et en particulier a la discussion portant sur les moulins à roue horizontale et à roue verticale. Les fouilles archéologiques, menées à Chypre au cours de ces dernières années, remettent en question le jugement reçu selon lequel on oppose la technique la plus simple de la roue horizontale a la technique plus complexe de la roue verticale, attribuée a l'occident et symbole de son avance technologique. Vincent Lagardere en étudiant les moulins d'occident musulman au Moyen Age rapelle l'origine orientale de ces deux roues, la raha horizontale, la saniya verticale et

${ }^{4} \mathrm{~F}$. THIRET, La RoInanie vénitienne au Moyen Age. Le développeınent el l'exploitation du domaine colonial vénitien XIle-XVe siècles, Paris, ed. de Boccard, 1959, p. 321, note 3 et pp. $417-418$.

SM.L. von WARtBuRG, Production du sucre de canne d Chypre: un chapitre de rechnologie médiévale. Cet article de 13 pages dactylographiées accompagné de 11 figures $\mathrm{m}$ 'a été obligeamment communique par l'auteur, il doit paraître en 1995 dans les actes du Colloque Domines le monde, ed. Annand Colin. 
soutient que le choix dépendait des conditions hydrauliques ${ }^{6}$. L'examen des installations de broyage à Chypre, a Couvoucle, à Kolossi et à Episcopi, prouve que les Latins ont utilisé une technique de roue horizontale connue du proche orient, où l'accroissement de l'énergie motrice s'effectue "grâce à un canal où s'engouffre l'eau produisant un jet à haute pression", un type qui "permit même la propulsion des meules lourdes en position verticale". Ces fouilles remettent par conséquent en question l'idée généralement acceptée du retard technologique des sucreries de la Méditerranée orientale, qui auraient été concurrencées victorieusement par les nouveaux centres basés sur l'utilisation de la roue verticale qui auraient été mis en place a partir de la seconde moitié du XIV ${ }^{\circ}$ siècle en Méditerranée occidentale. Mon objectif ici, à partir de l'étude de la culture de la canne à sucre en Espagne est d'apporter quelques éclaircissements a une étape encore mal connue du cycle de la canne et de la production du sucre dans l'occident méditerranéen. Les conditions de culture et de production industrielle a Valence apportent certains éléments pour apporter des réponses à ce débat.

En Méditerranée occidentale des études récentes sur la Sicile permettent de mesurer l'aire de diffusion de cette plante et les modalites originales de son exploitation. Au début du XIV ${ }^{e}$ siècle, c'est une plante de jardin que l'on trouve cultivée à Palerme, mais en très petite quantité. La phase d'expansion se situe entre 1360 et 1420 . La noblesse municipale en a pris l'initiative, elle conduit les operations de culture et de raffinage sur ses propres domaines en constituant des sociétés. Mais la culture est aussi le fait de petits entrepreneurs, ces derniers n'accomplissent pas le travail de raffinage, ils portent leurs récoltes aux moulins pour y recevoir leur part de sucre. La canne à sucre s'êtend dans l'île au nord, à l'est et à l'extrême ouest $^{7}$, après 1420 .

Dans le royaume de Valence en Espagne, l'implantation de la canne à sucre se développe en même temps qu'en Sicile. Les premiers témoignages de l'existence de plantations se situent sur des domaines de la plaine d'Oliva au sud de Valence, au début du XV' siècle. La culture de la

\footnotetext{
${ }^{\circ} \mathrm{V}$. LAGARDERE, Moulins d'Occident musulman au Moyen Age, IXe au XVe sizcles: Al Andalus, "Al-Qantara. Revista de Estudios Arabes", XIl-1 (Madrid, 1991), pp. 58-118.

${ }^{7} \mathrm{H}$. Bresc, Les Jardins de Palerine 1290-1460, Paris MEFrM tome 84-1 (1972), pp. 57 127. Un monde méditerranéen. Economie et Société en Sicile 1300-1450. 2 vol. Paris Rome, 1986, ed. de Boccard. I I pp. 223-252. Les jardins royaux de Palerme, Paris MEFRM tome 106-1 (1994), pp. 239-258.
} 
canne existe déjà en 1414, cette année la, le maître comptable du roi Berenguer Minguet constate, en effet, que les cannes à sucre que l'on fait pousser à Oliva rapportent de "gros émoluments". C'est à son incitation que le roi forme une société avec un changeur Francesch Scrivana, un marchand Johan Bayona et le maître sucrier Nicolau de Santa Fe pour créer une compagnie sucrière. Cette société se procure la canne à sucre dans les environs de Castellon de Burriana, la culture de la canne existait déjà dans cette région. C'est une plaine côtière de dixhuit kilomètres de long et de cinq a huit kilometres de large, elle est alimentée par le canal majeur de Castellon, ce dernier accomplit la prise de l'eau du Mijares à la confluence de la rivière et de la Rambla de la Viuda, il irrigue successivement les terroirs de Villareal, de Castellon, d'Almassora puis de Burriana. La première année la compagnie verse tout d'abord 600 florins pour se procurer les boutures de cannes a sucre des le mois de juillet 1415, sans que les sources précisent si les associés s'adressent à des ortolans ou à des paysans chrétiens et musulmans pour acheter ces cannes, de même on ignore quel est le mode de faire-valoir des terres adopté par la société. Castellon de Burriana est une terre de "realengo", dépendante du roi, sur ces terres les paysans disposent de baux emphytéotiques. Pour encourager cette culture de la canne, la nouvelle compagnie sucrière a-t-elle utilisée la formule du contrat d'association aparceria entre les propriétaires et plusieurs paysans associés avec partage de la récolte par moitié, ou la formule des contrats $a$ partido, forme d'association égalitaire entre deux ou trois associés avec partage de la récolte par moitié ou par tiers ${ }^{8}$ on ne sait pas comment étaient organisées les cultures sur les terres appartenant au roi et a la société. La compagnie débourse ensuite 1.000 livres pour édifier la maison du sucre et le moulin où vont s'accomplir les diverses opérations de raffinage.En décembre de la même année, ils déboursent à nouveau 1.100 livres pour réaliser des plantations de cannes sur le territoire de Castellon de Burriana. L'année 1417, ils effectuent des travaux dans la maison du sucre, dans les batiments d'exploitation du moulin, dans les divers celliers et hangars, le montant de la somme versé par les associés atteint sept cents

'E. AzNAR VAunejo, La integracion de las Islas Camarias en laoroma de Castilla (14781526) Aspectos administrativos sociales y economicos. Universidad de Sevilla. Universidad de la Laguna. Colleccion Viera y Clavijo $n^{\circ}$ VI, 1983, pp. 239-242 montre que les contrats a partido sur les champs de cannes aux Canaries adoptent la forme de l'association, de même le systeme de l'affermage existe pour les jardins irrigués et les champs de cannes, la rente se paye en nature. 
livres. A l'automne, en novembre 1418, avant la récolte et le début du raffinage, la société effectue une dépense globale de 4.380 livres. La société sucrière à cette époque a-t-elle étendu la superficie des champs de cannes exploités en concédant en affermage ou en complant de nouvelles terres à des paysans, a-t-elle augmenté de ce fait les capacités de raffinage du moulin et développé les infrastructures du complexe sucrier? Par la suite, cette dépense exceptionnelle se réduit en 1419-1420 à 2.000 livres. En 1421, le régent de la compagnie, Le changeur Francesch Scrivana meurt, le marchand Johan de la Torre lui succède à la tête de cette dernière. Au cours de deux ans pendant lesquels on suit encore les activités de la compagnie sucriere de Castellon de Burriana, les dépenses diminuent progressivement: 640 livres en 1421, 492 livres en 1422. Il faut mentionner ici que les investissements tixes ont été versés par les associés, ils donnent un fonds de roulement par la suite, mais la nature même de la source constitué par des documents concernant uniquement les dépenses de la compagnie sucrière ne permet pas de savoir ce que rapporte cette derniere. Après mars 1422, le maître comptable ne mentionne plus la participation du roi à la compagnie. A-t-elle été dissoute? Les plantations et le moulin avec la maison du sucre ont-ils été cédés ou rachetés par les associés du roi? La compagnie a-t-elle survécu après ces huit années d'exploitation, aucun autre indice ne permet d'en savoir plus'.

Néanmoins on voit qu'il s'agit de cultures qui ont persisté au nord de Valence dans la seconde moitié du XVe siecle et au XVI ${ }^{e}$ siecle. A l'occasion d'un litige entre l'Eglise et les paysans dans la zone irriguee du val de Sego, sur le territoire royal de la ville de Murvedro, les chrétiens et les musulmans se plaignent au souverain que l'évêque de Valence et le clergé cherchent indûmentà leur faire payer les dîmes des cannes à sucre qu'ils récoltent sur le terroir de Murvedro. Le roi Jean II ordonne au bailli de Valence de faire cesser ces exactions le 7 décembre 1461, les paysans devant la dîme et le tiers de dîme accoutumé aux collecteurs du roi ${ }^{10}$.

\footnotetext{
${ }^{9} A R V, M R ~ n 035$ fol 146 upoca du 17-4-1415 a 2-7-1415; MR n" 36 fol 150, 25-121415; MR 37 fol 197, 1417; MR 38 fol 237, 6-11-1418; MR 40 fol 314, 22-3-1421; MR 45 fol 279v., 7-11-1421; MR 42 fol 277.

${ }^{10}$ ARV, Bailia 1153 Lcttres missives. Calatuyud 7-12-1461 et Builia 1166 fol 303v.-304r.v. E. Ciscar Pallares, Tierra y Señorio en el Pais Valenciano (1570-1620), Valence, Del Cenia al Segura 1977, p. 103 note 71, constate que sur les ćtats du duc de Segorbe, en 1577, les musulmans acquittent une part symbolique de la production de sucre.
} 
Si la culture de la canne a existé précocement dans la plaine de Castellon, à Segorbe et à Murviedro, si cette culture se poursuit à Segorbe jusqu'au dernier du XVI ${ }^{e}$ siecle, la grande région de production des cannes se situe sur les terres irriguées au sud de Valence, celles de Gandia et d'Oliva. C'est une région de domaines seigneuriaux, ils disposent d'une abondante main d'oeuvre de paysans musulmans redevables de corvées en travail. La plaine côtière est irriguée par le canal royal d'Alcoy qui dessert Gandia et Oliva, la prise d'eau se situe a partir du fleuve Serpis et du barrage d'Alcoy" ${ }^{11}$. Dans ces jardins irrigués a la longue tradition horticole, l'introduction des plants de cannes bénéficie du savoir faire des paysans musulmans et chrétiens, qui peuvent réaliser toutes les tâches nécessaires depuis la plantation par bouturage à la récolte des cannes la seconde et la troisieme année du cycle.

Les contrats notariés souscrits entre 1474 et 1513 au nombre de trente cinq concernent surtout des affermages de domaines et de moulins sucriers de la noblesse locale à des marchands entrepreneurs. Les affermages ont lieu à l'automne en général, ou en novembre avant le début des opérations de cuisson. La communauté musulmane de la seigneurie où est implantée le moulin consent avant le contrat a la vente d'un cens sur l'alja$m a$ afin de pouvoir acheter le bois nécessaire à alimenter les chaudières, de recruter la chiourme salariée qui travaille au moulin pendant quelques mois et de pourvoir à toutes les nécessités du moulin. Dans un second temps le seigneur et son épouse s'engagent avec un marchand entrepreneur à observer des chapitres souscrits devant le notaire pour une durée de un an à quatre ans. La formule choisie consiste souvent à assurer deux ans d'exploitation "ferme", on peut la reconduire pour deux années dites de répit, que le fermier notifie par lettre au seigneur en janvier de la seconde année d'exercice. Au moment de l'élaboration de l'acte, un chapitre décrit souvent avec minutie l'équipement du moulin. Sur le domaine de don Johan de Cardona, seigneur du Real, le moulin se compose de douze meules (rotlons), de six presses (premses), et de dix huit chaudieres (calderes) et de tous les instruments nécessaires. Il est entendu que, si au cours de l'utilisation, un instrument de cuivre, une meule ou une presse se rompent, il revient au fermier d'effectuer les réparations à ses frais, mais si avant

"T.F. Guck, Irrigation and Society in Medieval Valencia, Harvard Universily Press Cambridge Massachussels, 1970, pp. 206-216. 
l'exploitation le moulin nécessite un entretien, la dépense en revient au seigneur. A son entrée en charge, le fermier doit faire le compte de tous les instruments indispensables à la cuisson du sucre. Il tient un inventaire écrit de sa main, dont il adresse un double au propriétaire, ces documents sont souscrits par les intéressés. Don Johan de Cardona reconnaît que sur les dix huit chaudières dont dispose son moulin, quatre nécessitent un étamage qu'il est impossible de réaliser au cours de la première année du contrat, car le temps de la cuisson du sucre approche. On convient qu'elles seront étamées l'année suivante aux frais du bailleur, de même sur les douze meules, une s'avere inutilisable, le seigneur est tenu de la remplacer à ses frais, sinon le fermier veille à effectuer la réparation et à déduire de son compte les dépenses qu'il assumera. L'ensemble des six presse se présente en bon état de marche, le preneur doit les rendre comme il les a prises. Si toute l'attention se concentre sur le moulin, sur la machinerie, sur les bâtiments d'exploitation, on ne néglige pas pour autant la pérennité du cycle des plantations.

A l'issue du contrat, la dernière année le preneur s'engage à laisser autant de plants pour le bouturage qu'il aura récolté de cannes, mais si à cette époque il a planté quelques champs de cannes de première année, encore impropres à la "cuisson", il doit les laisser sur place pour que son successeur puisse faire accomplir le marcottage.

Si l'on admet l'opinion dominante selon laquelle les paysans disposent de tenures emphytéotiques, dans la zone irriguee un quart de la surface de leurs champs était encore consacrée à la culture de la canne à sucre au $\mathrm{XVI}^{\circ}$ siecle, mais on peut former l'hypothèse qu'au moment de la pleine expansion de cette culture au $\mathrm{XV}^{\circ}$ siecle les plantations de cannes occupaient une superficie plus étendue. Leurs redevances en nature portaient sur la moitié de la récolte des cannes et ils vendaient l'autre moitié leur appartenant au moulin ${ }^{12}$.

On peut éventuellement penser que les techniques horticoles évoquées à la fin du XVIII ${ }^{\circ}$ siecle ne sont pas trop éloignées de celles qui se pratiquaient aux $\mathrm{XV}^{-}-\mathrm{XVI}^{\circ}$ siecles. Lorsque le géographe Antonio Jose Cavanilles parcourt la huerta de Gandia a la fin du XVIIIe siecle, il constate que la canne est encore cultivé à Benirredra, à Benipeixcar et à Gandia.

\footnotetext{
12J. CASEY, The Kingdom of Valencia in the seventeenih century, Cambridge 1979, pp. 84, 106-107.
} 
Il décrit minutieusement les différentes phases de la culture, la plante se reproduit avec des bouture de cannes de l'année antérieure, qui doivent présenter trois noeuds ou plus et atteindre une paume de large. On les conserve de deux manieres, soit en les enterrant dans un champ, soit en les recouvrant de feuilles dans un endroit abrité. Il est nécessaire de les remuer tous les quinze jours pour qu'elles ne fermentent pas. Fin mars, les laboureurs préparent la terre en procédant à de multiples labours, sur chaque portion de champ de 64 ares, il faut déverser neuf tonnes de fumier au cours de la plantation. Les paysans divisent les champs en bandes (trofas), de six à sept paumes paralleles entre elles, ils laissent vides cinq paumes; dans la section d'une paume et demie restante, ils tracent des lignes transversales distantes d'une paume, sur chaque ligne transversale, ils plantent quatre cannes en laissant entre chacune deux doigts. A la fin du mois de mai, grâce aux arrosages pratiqués au pied de chaque bande, les jeunes pousses atteignent un pied de hauteur. Commes les cannes ne disposent encore que de très courtes racines au cours de ces deux mois, le cultivateur utilise les espaces vides de la bande de terre pour élever en culture dérobée des choux ou des salades que l'on arrache à la fin mai. Au début du mois de juin, on creuse à la bêche la terre entre les rangées de cannes afin de déposer du fumier que l'on recouvre avec cette terre. Au fur et à mesure que les cannes poussent, on ajoute la terre de la bande de culture dérobée, on enterre la partie inférieure des cannes, on fait passer l'eau d'irrigation par la rigole que l'on a formée. Quand les cannes atteignent 2,70 metres, on les coupe en novembre, elles forment alors une forêt de 120 cannes par trofa de $7,8 \mathrm{~m}^{213}$.

Même si l'échelle de culture a changé. Il est probable que la densité des plantations était plus grande et peut-être le rôle des cultures dérobées moindre. Pendant toute la période de la récolte, le fermier dispose de gardes sur les plantations, ces derniers doivent veiller à ce qu'aucun paysan ne soit en mesure de pouvoir voler des cannes pour son compte propre. Il est stipulé dans les contrats que le seigneur délègue le droit de basse justice qu'il exerce sur ses hommes au marchand entrepreneur. Le preneur peut user de ce pouvoir en infligeant des peines pécuniaires aux paysans. En

\footnotetext{
${ }^{13} \mathrm{H}$. Bresc évalue à 96 à 270 cannes par carreau de $5,5 \mathrm{~m}^{2}$ leur densité en Sicile. Un Monde méditerranéen, 1, p. 229; A.J. CAVANIUES, Observaciones sobre la Historia natural, Geografia, Población y Frutos del Reyno de Valencia, Madrid, Imprenta Real, 1795, t. 1, pp. 105,216 ; t. 2, p. 142.
} 
vertu du droit de commandement sur les hommes de la seigneurie que le seigneur lui a transmis, le marchand Francesch Desparça a la possibilité de contraindre les paysans musulmans et les chiourmes de journaliers agricoles, engagés au moment de la récolte et du raffinage à l'accomplissement du travail nécessaire au fonctionnement du moulin. Il peut exiger ce devoir en recourant à la force et même, si le besoin s'en fait sentir, en privant de liberté les récalcitrants, étant entendu au moment de la rédaction de l'acte, que les amendes de justice lui restent acquises.

A propos des phases de l'élaboration du sucre, les contrats sont peu prolixes. On voit mentionner les rouleaux, dans le moulin du Real, on fournit au preneur douze meules et six presse ou rouleaux.

Ces presses se composent de trois cylindres disposés horizontalement ou verticalement, actionnés par l'eau ou par la force animale. Ce sont les presses qu'il faut soigneusement nettoyer après chaque usage. En effet il est stipule qu'apres chaque phase de cuisson, le fermier doit faire nettoyer les presses en les curant et en les "creusant pour qu'elles conservent la forme accoutumé". Je suppose qu'apres avoir subi une operation de concassage, les cannes sont broyées par les meules des moulins. On ne connaît pas les dimensions de ces meules, mais à la suite de fouilles menées a l'emplacement d'anciennes sucreries au Maroc, on a trouvé des meules d'1,20 metre de diametre, de 45 centimetres de large avec un évidement axial de 20 centimètres $\mathrm{Ce}$ sont des dimensions proches que l'on retrouve dans les moulins sucriers de Sicile de 1,31 metre à 1,50 metre de diametre, pour 50 centimetres de large ${ }^{14}$. Au Real, les six presse à rouleaux alimenteraient soit six moulins fonctionnant avec deux meules ou quatre moulins broyant avec trois meules comme on le remarque sur une gravure d'epoque de Jean Stradan représentant la fabrication du sucre. Les opérations de raffinage se déroulent dans le "sostre" le hall couvert. Marie Louise von Wartburg a décrit le plan de la maison du sucre a Couvoucle (Chypre). Les foyers étaient séparés des chaudieres pour éviter que la cuisson des sucs ne puisse être troublée et souillée par les cendres des feux. Au Real les dix huit foyers alimentent les dix huit chaudieres de cuivre, cette installation est très importante si on la compare aux autres installations établies dans les moulins sucriers valenciens.

${ }^{14} \mathrm{P}$. BE:RTHIl:k, Les anciennes sucreties au Maroc et leurs réseciux hydrauliques, Rahbat 1966, 2 vol., vol. 1, p 143. 
Les comptes de la duchesse de Gandia, María Enríquez de Borja permettent de reconstituer utilement l'ensemble des opérations de cuisson $^{15}$. Les premières chaudières sont celles qui reçoivent le jus de canne en sortant du bac citerne où il est conservé peu de temps, car les vesous ont tendance à fermenter rapidement. On commence à purifier le jus en y ajoutant de la cendre et de la chaux. Ce jus est tamisé au travers d'étoffes de laine ou de tamis de métal au moment de le faire passer dans d'autres chaudières. Au cours de la troisième cuisson on jette dans le vesou des produits végétaux pour enlever les dernières impuretés prélevées à l'aide d'écumoires en cuivre. Lors de la quatrième opération le vesou est soumis à un feu plus vif, il se réduit aux trois quarts. C'est le moment où après l'arrêt des foyers, on le transvase dans de grands récipients troués au fond, ceux-ci laissent à leur tour s'écouler le liquide sucré dans des pots en terre cuite évasés au sommet, étroits du bas, les formes de sucre ${ }^{16}$, élaborés souvent sur place par des potiers musulmans ou chrétiens.

Ces operations ont lieu dans la maison du sucre disposant de son "engutador" et de son "scurador", de son égouttage et de son canal d'écoulement, quant aux résidus, ils subissent une nouvelle cuisson et constituent une sorte de miel de cannes ${ }^{17}$. La séparation du sucre solide et du sirop par l'égouttage et le clairçage débouche ainsi sur la production de sucres candis, si le sirop a été soumis à une évaporation lente et spontanée. Si l'évaporation a été plus rapide et que le mélange a été agité, battu, on obtient de petits cristaux plus ou moins agglomérés. La mélasse de sucrerie, c'est-à-dire le dernier sirop de cristall isation se caractérise quant à elle par une quantité notable de sucre en dissolution dans une proportion d'eau relativement faible. En examinant le plan idéal d'une sucrerie au nouveau monde au début du XVIII' siecle, on constate que les chaudières de cuivre dans lesquelles on cuit le sucre, portent des noms qui symbolysent les différentes opérations de cuisson des vesous: la grande Chaudière, la Pro-

\footnotetext{
${ }^{15}$ AHNM, Osuna 1170 (15), 5-8-1497 Trapig du lieu de Peresa.

${ }^{16}$ AHNM Osuna 1170 (10) 4-9-1490 achat par Francesch de Roquamora, procurcur du duc Jean de Borja de 2.000 fornes de sucre à 13 I 2 s le millier, à Cahat Madrella de Vilalonga. ARV P 2020, 20-7-1503 Pasqual Codori de Manises vend à Johan Daguilera 2000 fonnes de sucre à 81 le millier.

"On retrouve ce processus en Egyple. J. MAZUEL, Le sucre en Egyple. Elude de géogrphie historique el économique, Le Caire. Publications de la société royale de géographie d'Egypte 1937, pp. 9-16, 23. R.J. Foril:S, Studies in ancient technology. vol. 5. Leiden 1957. G. ESTORD, L'industrie du sucre de canne, Paris 1958.
} 
pre, la Lessive, le Flambeau, le Sirop, la Batterie ${ }^{18}$. Dans la dernière, on doit battre le mélange avec une écumoire pour lui donner de l'air, afin de l'empêcher de se répandre. Les chaudières diminuent de diametre et de profondeur au fur et à mesure qu'elles sont proches de celle où le sucre reçoit sa dernière cuisson. Déjà au $\mathrm{XV}^{e}$ siècle, lors d'un contrat énumérant les droits sur le moulin de Peresa, de María Enriquez de Borja, duchesse de Gandia, on constate que ce trapig est de moindre dimension que celui du Real appartenant à don Johan de Cardona. L'inventaire fait état de quatre meules, de trois presses et de huit chaudieres. Elles sont décrites selon l'ordre des différentes phases de chauffe des vesous ${ }^{19}$ : "calderes de parop, de colar, para fer lent, de coure". Les deux premieres correspondent a la phase de la Chaudiere et de la Propre, la troisième sert a la lessive, les quatre autres aboutissent au sirop, la dernière est appelée la chaudière à cuire proprement dite. L'intérêt de ce document est de mentionner en outre des objets de métal que l'on regroupe ailleurs sous le terme global "d'instruments nécessaires au trapig". On énumère la présence de deux barres de fer, d'une fourche de fer, de trois cuves, de quatre seaux, de deux bassines, d'une louche pour extraire la pâte, de deux écumoires et de deux tamis indispensables pour mener a bien les phases finales de la lessive et de la batterie.

Le coût de ce matériel est difficile à évaluer, néanmoins en 1481, un contrat d'achat de huit chaudieres destinées à équipper le moulin de Beniarcho, appartenant au chevalier Johan Tolsa de Ripoll permet de connaître le prix de ces récipents de cuivre qui atteignent un montant de 241 livres 1 sou 11 deniers, l'achat se réalise par l'intermédiaire du marchand Pere Siurana ${ }^{20}$. Au début du XVI $I^{e}$ siecle ce cout paraît moins élevé, peutetre parce que le duc de Gandia s'adresse directement à un chaudronnier, le montant de huit chaudieres ne depasse pas alors 206 livres 18 sous 8 deniers $^{21}$. On a vu que les foyers a feux vifs placés à l'extérieur du bâtiment où l'on raftine les sucs consomment d'énormes quantités de bois de chauffe. Il semble que dans la premiere phase d'exploitation de la canne on ait puisé ce hois de chauffe sur les premieres pentes des massifs montagneux

\footnotetext{
18.B. LABAT, Nouveaux vo yages aux illes de l'Amérique, III, Paris 1722, pp. 176, 254.

${ }^{19}$ AHNM Osuna 1170 (15), 5-8-1497.

${ }^{20}$ AHNM Osuna 1350 (2), 1-10-1481.

${ }^{21}$ AHNM Osuna 1171 (1), 16-12-1501.
} 
dominant les valllées et les plaines irriguées. Pourtant les autorités royales s'en inquietent, en janvier 1442 , le bailli général de Valence adresse une lettre au bailli de la ville de Corbera au sud du Jucar, il a appris que le déboisement entrepris par les maures et les chrétiens se réalise aux dépens des oliviers, des caroubiers, et d'autres arbres de la région, pour alimenter les foyers des moulins à sucre du val d'Alfandech. On perçoit ici comment l'extension de la culture de la canne se fait aux dépens de cultures hautement spéculatives comme celle de l'olivier, on peut s'imaginer également quel bouleversement elle a fait subir à toute une économie agricole existante, les caroubes servant à l'alimentation du bétail, c'est tout un écosystème assez fragile que la canne à sucre détruit dans cette phase d'expansion. En Sicile on assiste à un phénomène similaire, les paysans pour se procurer le bois ont recours à l'oléastre, mais aussi à l'yeuse et au chêne liège. Le déboisement dans les premiers massifs surplombant la plaine de Gandia et d'Oliva s'est-il poursuivi avec toutes ses conséquences ou les mesures de sauvegardes, assorties d'amendes, prises par les autorités royales ont-elles été respectées? Il n'est plus fait mention par la suite des essences locales, les contrats de ventes de bois de chauffe aux moulins dans les dernières décennies du $\mathrm{XV}^{\bullet}$ siècle portent sur la vente de bois de pin, provenant des terres du marquisat de Moya, des lieux dits de Cafrilla, Cirueles, Pos de la Campana, les bois sont charriés vers la Rambla d'Ademuz jusqu'au Guadalaviar-Turia, pour être acheminés en novembre à partir de la fête de la Toussaint. Les achats annoncent des quantités qui oscillent entre 1000 et 2000 quintaux de bois, c'est-à-dire entre 50 et 100 tonnes. La dépense atteint 88 livres 10 sous 8 deniers en 1481 pour 50 tonnes, elle est de 141 livres pour 100 tonnes en $1495^{22}$. A chaque fois les acheteurs sont des marchands entrepreneurs engagés dans l'affermage et l'exploitation des moulins sucriers seigneuriaux. Si l'on cherche à savoir ce que représente le cout du travail dans les dépenses d'exploitation, on dispose de quelques éléments à partir de comptes de la duchesse de Gandia concernant les trapigs de Bellreguart, de Miramar et du Real. Le maître sucrier musulman

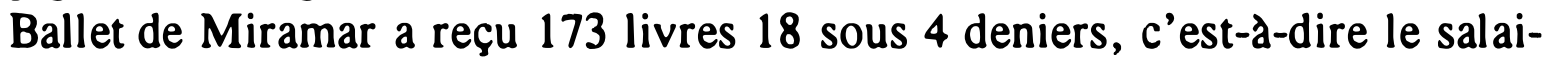
re de deux années un mois et vingt trois jours, période au cours de laquelle il a oeuvré dans la raffinerie "refinador" et le moulin "trapig" de Gandia. Pour la même durée son "négre" qui l'a assisté dans sa tâche reçoit la

${ }^{22}$ ARV P 2000, 25-5-1481. P 2011, 16-1-1495. 
somme de 9 livres 7 sous 6 deniers. Ils ont oeuvré ainsi à partir du 7 avril 1498 à la fin mai 1500. Ailleurs les paysans musulmans perçoivent un salaire calculé sur le temps de travail d'une année. En 1503, pour le trapig de Bellreguart, le salaire de Barberus atteint cinquante livres, ce qui est considérable en comparant cette somme aux salaires annuels que reçoivent des musulmans du trapig de Miramar, oscillant entre 10 et 20 livres entre 1503 et 1504, ou aux 15 livres annuelles de Mahomat Bayaya au moulin du Real ${ }^{23}$. Barberus devait certainement assurer les fonctions de maître sucrier a Bellreguart, la somme qui lui est remise en juin 1503 ne représenterait qu'une partie de son salaire, alors que les salaires des autres musulmans travaillant au moulin et a la raffinerie varient entre quinze et vingt livres annuelles. Les paysans devaient être aussi rétribués en produits du moulin sucrier, mais ils devaient aussi recevoir sous cette forme le montant de la vente de leur récolte de cannes qu'ils portaient au trapig. C'est ainsi qu'à la suite d'une saisie de biens de quelques musulmans de l'"alqueria" de Buxerques dans le val de Vilalonga, lors de l'inventaire des effets et meubles de la maison d'Abrahim Boamit, on relève la présence de huit formes de sucre pleines. Cette mention est un indice de la participation des musulmans a la commercialisation des sucres qu'ils reçoivent soit comme complément de salaire, soit comme le prix de la vente de leur propre récol$\mathrm{te}^{24}$.

Le travail servile n'apparaît pas, on peut se demander quel est le statut de l'homme qui assiste le maître sucrier musulman à Miramar, désigné dans le document comme "son negre", il reçoit néanmoins un salaire annuel de 4 livres 17 sous 3 deniers, soit un peu moins de la moitié du plus bas salaire perçu par les musulmans de Miramar, mais il n'est pas désigné comme étant un esclave. Au moment de la récolte et de la production du sucre, des équipes de journaliers agricoles sont engagées et rétribuées. Le travail sala rié des chiourmes des moulins permet ainsi d'épauler les paysans des seigneuries en organisant des tours de roulement et des équipes de travail, puisque.les opérations de cuisson peuvent s'effectuer jour et nuit au moment du raffinage. La seule mention de la présence d'une esclave est la déclaration d'un maître sucrier Johan Chrestia devant le bailli général de Valence en février 1480. Ce dernier dénonce la fuite d'une

\footnotetext{
${ }^{23}$ AHNM Osuna 1171 (2), 30-5-1503, 2-6-1503, 5-12-1503, 23-12-1503, mars 1504.

${ }^{24}$ AHNM Osuna 1168 (8), 17-1-1474.
} 
esclave blanche musulmane agée de vingt trois ou de vingt quatre ans, du trapig qu'il possede dans la ville de Gandia ${ }^{25}$. Sur ce probleme, je rejoindrai la position de Marie Louise von Wartburg qui n'a trouvé à Chypre, dans la Chronique de Léonce Macheras qu'une seule allusion à un esclave musulman converti, maître sucrier qui fabriquait des poudres de sucre et des sirops en 1426. A Valence, dans les dernières décennies du XV' siècle, la seule mention de travail servile concerne une esclave blanche musulmane en fuite, qui devait être une esclave domestique appartenant à Johan Chrestia ne travaillant ni au moulin, ni dans la raftinerie. La culture de la canne, le raftinage restent ici comme a Chypre et en Sicile entre les mains des paysans musulmans ou chrétiens des seigneuries, louées et affermées par les marchands entrepreneurs.

La commercialisation du sucre constitue une autre étape. Il faut distinguer la négociation des ventes globales des récoltes de la vente sur une moindre échelle. Les ventes globales ont lieu à la fin de la période dite "de cuisson", elles sont négociées soit par les seigneurs détenteurs des moulins et des raffineries ou par l'intermédiaire de leurs procureurs, soit par les représentant des musulmans du lieu où fonctionne le trapig. Ces ventes conclues habituellement de février à août, stipulent qu'il s'agit de livraisons évaluées selon l'unité de mesure et selon la qualité du produit: la charge de sucre de 140 kilogrammes environ, le "canter" ou "viceo" de mélasse de 4 litres, enfin les formes pleines de sucre "fin ou batard". Les contrats les plus importants font état de la production d'un ou de plusieurs moulins à sucre. En 1488, à Gandia les chevaliers Jacme et Johan Balaguer promettent de livrer 28 tonnes de sucre à deux maîtres sucriers de Valence pour la sommes de 2.680 livres $^{26}$. Dans la même région, le trapig de Bellreguart appartenant au duc de Gandia produit 21 tonnes de sucre pour un montant de 1.725 livres $^{27}$. Cette production peut être plus réduite ou plus importante. Au début du XVI I $^{e}$ siecle, le petit trapig de la Palmera appartenant au chevalier Guillem Ramon Pujades ne produit que 14 tonnes de sucre $^{28}$. Par contre au cours de l'année 1512-1513, les différents trapigs du duc Jean de Borja atteignent une production de 86 tonnes qu'il négocie

\footnotetext{
${ }^{25}$ ARV Bailia 1156, fol 716, 5-2-1480.

${ }^{26}$ ARV P 2005, 18-3-1488 et 26-3-1488 a 13110 s la charge.

${ }^{27}$ ARV P 2007, 2-5-1491 a 11110 s la charge.

${ }^{28}$ ARV P 2022, 6-5-1506 à 11 I la charge pour une somme de 1100 livres.
} 
pour une somme de 6.037 livres 10 sous $^{29}$. Très souvent les acheteurs se rendent acquéreurs des formes de sucre pleines. En 1499, Rodrigue de Borja, seigneur de Vilalonga vend 920 formes de sucre réalisées dans son trapig de Rafol, la même année les trapigs du comte d'Oliva Ramon Riusech fournissent 1800 formes $^{30}$. Cette production s'intensifie de maniere considérable de 1500 à 1503 , pour passer de 2.980 formes en 1500 , a 3.622 formes en 1501 , puis a 3.728 formes en 1502 , elle culmine a 4.662 formes en 1503. Au cours de cette dernière année, le procureur du comte d'Oliva reçoit la somme de 8.807 livres 3 sous pour cette derniere ven$\mathrm{te}^{31}$. Pendant ces trente décennies, les prix de la forme de sucre se maintiennent de 22 à 23 sous l'unité, une seule fois le prix s'abaisse en 1499 à 16 sous 6 deniers.

Plus rarement les contrats font état des différentes qualités de sucre réalisé. En 1490, un contrat stipule les quantités de "mesurades" et de "remelles", c'est-à-dire de résidus et de miels de cannes que l'acheteur s'engage à prendre; elles représentent respectivement $2,5 \%$ et $11 \%$ des sucres. Leur prix de vente est évidemment beaucoup plus bas, celui des "mesurades" s'abaisse a 13 sous la forme, celui des "remelles" a 4 sous 6 deniers ${ }^{32}$.

Il est plus difficile de mesurer la commercialisation des mélasses, car les évaluations sont imprécises par nature. Le représentant des maures du trapig de Vilalonga et de Potnes promet a Luis Manrana de lui vendre toutes les mélasses qu'ils produiront en ce lieu ${ }^{33}$. Gaston de Moncada emploie la même formule auprès du marchand Jacme Macip, lorqu'il s'engage a lui donner pendant sept ans toutes les mélasses de son trapig de Beniargo $^{34}$. Ce marchand réussit par la sui à partir de 1507 à s'assurer pour quatre ans la totalité de la production des mélasses de la duchesse de Gandia, a Gandia ${ }^{35}$. On constate que le prix de vente du "viceo" de mélas1513.

${ }^{29}$ AHNM Osuna 1171 (5), 5-7-1512. Osuna 1171 (6), 13-1-1513. ARV P 2030, 28-1-

${ }^{30}$ AHNM Osuna 1308 (9), 1-3-1499, 9-3-1499. ARV P 2017, 25-8-1499.

${ }^{31}$ ARV P 2018, 2-6-1500. P 2019, 1-4-1501. P 2011, 24-2-1504.

${ }^{32}$ AHNM Osuna 1170 (10), 19-5-1490.

${ }^{33}$ ARV P 2007, 26-2-1491. A 9 s par viceo ou canter

${ }^{34}$ ARV P 2022, 5-11-1505. A 1 s par viceo.

${ }^{35}$ ARV P 2024, 15-1-1507 a 2 s par vicco. 
se a doublé de 1505 à 1507 , il semble que la demande ait été plus forte alors. On peut se demander si la multiplication des ventes de mélasses ne signifie pas déjà dans les dernières décennies du $\mathrm{XV}^{\mathrm{e}}$ siècle et au début du $\mathrm{XVI}^{\circ}$ siecle, un accentuation du probleme posé par les besoins en bois de chauffe et l'impossibilité pour les raffineries valenciennes de procéder à la totalité des étapes du raffinage pour l'ensemble de la production. La seconde hypothèse peut conduire à s'interroger sur la manière dont on utilise la mélasse exportée. Est-elle raffinée ailleurs pour en retirer encore du sucre, sert-elle de base à certaines préparations de confiseries et de patisseries, ou est-elle distillée après fermentation pour être transformée en alcool? Ce produit exporté par barils essentiellement vers l'Europe septentrionale peut correspondre à un type particulier de consommation propre à cette zone des sous produits de cuisson.

Les marchands valenciens et les maîtres sucriers sont les acquéreurs de la production des trapigs. Il leur revient ensuite de devenir les intermédiaires auprès des marchands étrangers et des compagnies. Lors de cette étape, les principaux acheteurs dans les dernières décennies du siècle sont les marchands flamands Pierre et Antoine Plener, les allemands de la Grande Compagnie de Ravensburg, la Petite Compagnie de Conrad Ancharita de Ravensburg, celle d'Henri d'Espagne alias Erimelli. A partir des années 1495, les marchands français aftirment leur présence lors de ces contrats d'achat. La compagnie avignonnaise de Bernat Maça, la compagnie de Jehan Polit et de Pierre Bertu de Crémieu en Dauphiné, les compagnies de Jacques Malivern et d'Imbert Rey, de Jehan Monret et de Philibert Serri de Bourg en Bresse, sont celles dont les noms reviennent le plus souvent a l'époque des expéditions de sucre à l'automne. La plupart des facteurs de ces compagnies présentes sur la place de Valence remboursent leurs achats de sucre ou de mélasse en cédant des créances équivalentes sur des revendeurs au détail, auprès desquels ils écoulent les marchandises qu'ils importent sur place. Les prix pratiqués alors sont deux fois à deux fois et demie supérieurs aux prix d'achat de départ auprès des trapigs. La charge de sucre vendue au trapig ou livrée a 12 ou 13 livres atteint 32 livres la charge, lors des achats réalisés par des étrangers auprès des intermédiaires valenciens. On distingue selon la qualité le sucre fin du sucre "batard", il y a toujours une différence de prix de 2 deui liv'res e.tre les deux types de 
sucre $^{36}$. Les expédition de sucres et de mélasses s'effectuent par voie maritime, entre 1478 et $1513,82 \%$ des contrats de nolisement à partir des ports de Valence, de Denia ou de Xavea ont pour destination la Flandre, l'Ecluse, la côte de Bruges, la Zélande. $18 \%$ des départs s'effectuent vers la Méditerranée: les rivages de Collioure, Aygues Mortes-Port de Bouc, I'ensemble Genes-Savone, Porto Pisano, Civita Vecchia- Riparoma, et enfin Naples. Comme on l'a souligné les chargements en direction de la Flandre et de l'Europe septentrionale se composent de barils de mélasses. Vers les ports méditerranéens, on expédie exclusivement des caisses de sucre. Cette opposition entre des amateurs de mélasses et les amateurs de sucre dur, estelle due à des habitudes de consommation différentes ou à des impératifs technolögiques, les operations ultimes de raffinage pouvant s'effectuer à l'arrivée dans les ports septentrionaux, ce qui n'était pas le cas dans les autres villes portuaires méditerranéennes évoquées ci-dessus.

Le marché du sucre valencien cependant, a la fin du $X V^{e}$ au début du XVI ${ }^{e}$ siecle, commence à subir la concurrence des plantations sucrieres creées dans les îles à Madere et aux Canaries par les Portugais et les Espagnols. La première mention d'arrivee de sucre de Madere date d'août $1492^{37}$. Or c'est à cette époque que les estimes des fonctionnaires royaux portugais évaluent la production sucrière de l'île à 1.045 tonnes, pratiquée par 221 producteurs, a travers une culture réalisée en régime de petite et de moyenne propriéte $e^{38}$. Cette classe de moyens et petits propriétaires aux possibilités limitées ne participe pas au négoce des sucres qui est entre les mains des marchands étrangers, italiens essentiellement. C'est par l'intermédiaire des marchands italiens installés à Valence Cesar de Barzi, Julian Pitti, la compagnie sièrfnoise des Spannochi que s’acheminent les premieres cargaisons de sucre de' Madere au prix de 16 livres la charge entre 1492 et 1503, au moment où Ye sucre de Valence se vend a 24-25 livres la char$\mathrm{ge}^{39}$. Les mentiôns de l'arrivée à Valence des sucres en provenance des

\footnotetext{
${ }^{36}$ Le prix de la charge de sucre fin varic entre 25 et 28 l, le sucre hatard est vendu entre 23 et 261, les mélasses sont contenues dans des barils de 64 litres, 1 haril $=16$ canters, vendus l'unite 22 s 6 d. ARV P 1999, 13-8-1479. P 2004, 3-12-1485.

${ }^{37}$ ARV P 2009, 27-8-1492.

${ }^{38} \mathrm{~V}$. RAU-J DE MACEDO, $O$ acucar da Madeira nos fins do século XV. Problemas de Producao e Comercio. Ed. Junta Geral do Distrito autonomo do Funchal 1962, pp. 11-37.

${ }^{39}$ ARV P 2009, 27-8-1492. P 2018, 7-1-1500. P 2019, 12 et 14-1-1501, 28-4-1501, 14 ct 19-5-1501. P 2020, 14-1-1503.
} 
Canaries commencent au cours de l'année 1501. Elles sont liées au fait que deux marchands génois de Valence Francisco Palomar, Benedetto Pinelli possedent des moulins sucriers de "l'île de Canaria"40, dont ils vendent une partie de la production a partir de 1500-1501. Il s'agit d'abord de 600 caisses de sucre à 14 livres la charge. En 1504, ils vendent à Bernat Catala 500 charges de sucre par an pendant cinq ans dès l'année 1504, c'est-à-dire 70 tonnes de sucre, que viennent chercher chaque année aux Canaries trois personnes emportant avec elles les "arroves" de Valence, soit une quantité un peu moindre que les 86 tonnes de sucre produites en 15121513 par les trapigs du duc de Gandia. Les envois s'effectuent à partir des Canaries en mai et en septembre, mais les prix très bas consentis de 12 livres 10 sous la charge à partir de 1504 jusqu'en 1509, même en ajoutant les frais de nolisement permet de concurrencer victorieusement le sucre local.

On peut imaginer les réactions des nobles qui étaient impliqués dans la production locale et aussi celle des paysans. Il semble que leurs suppliques aient été entendues puisque dans une politique contradictoire, car certaines de ces îles faisaient aussi partie de la Couronne, pour empêcher cet aftlux de sucre à bon marché, la monarchie adopte une politique protectionniste afin de garantir le marché en interdisant l'importation des sucres étrangers. Mais il semble que la tentation d'introduire des sucres livrés a bas prix, pour les revendre a un prix supérieur, sans payer les droits dus au roi et a la ville, en les faisant venir par les ports moins surveillés situés au sud, ait été grande ${ }^{41}$. En février 1513, le régent du baiIliage de Valence Johan Cavaller déplore les fraudes qui s'effectuaient au détriment des producteurs valenciens et des droits perçus sur le commerce. Il dénonce l'introduction de sucres fabriqués aux Canaries et dans "d'autres parts" par le port d'Alicante, il donne mission a Johan Fabregues de parcourir les villes et les chemins de ce ressort pour tenter de retrouver les

\footnotetext{
${ }^{40}$ Voir sur l'introduction de la culture de la canne aux Canaries et les fonnes d'exploitation en moyennes et petite propriétés de 7 a 14 ha, et de 19 hectares pour ceux qui s'engageraient à édifier un moulin sucrier E. AZNAR VALLEO, La insegracion de las Islas Canarias en la Corona de Castilla, pp. 229-250 et 260-264.

"ARV P 2013, 18-1-1496. Lettre testimoniale sur les instances de Johan de Rovres, naturel de Castille. Sur l'achat de sucre on paye à Valence: droit du general 9 d par livre, sisa 4 d par livre, quema 3 d par livre, péage 2 s par charge; pour frens e selles: droit du general $6 \mathrm{~d}$ par livre, sisa $4 \mathrm{~d}$ par livre, quema $3 \mathrm{~d}$ par livre, albara du bailli $1 \mathrm{~s}$.
} 
sucres prohibes et d'infliger aux contrevenants une amende de 500 florins$\therefore$. Devant la disparité des coûts, devant les problemes écologiques que rencontrait la culture du sucre, force est de constater que la belle époque du sucre de Valence s'achevait et que de nouveaux cycles de cultures allaient hientôt remplacer les plantations de cannes.

\section{RÉSUMÉ}

Le propos de cette contribution est de présenter les conditions qui ont prévalu lors de l'essor de la culture de la canne à sucre et de sa transformation dans le royaume de Valence aux $X V^{e}-X V I^{e}$ siecles. Cette analyse est hasée sur des sources inédites extraites des archives royales et de documents notaries, concernant les compagnies exploitant les moulins sucriers, les contrats entre seigneurs et fermiers, ce travail révèle le rôle crucial que les communautés musulmanes locales ont joué dans ce cycle. Il montre aussi comment la concurrence du sucre des îles atlantiques amène le déclin détinitif de cette culture à Valence. En discutant les problèmes de technologie et de rapport de production, il est fait référence à des questions soulevés par l'historiographie sur la production sucrière en Méditerranée.

\section{SUMMARY}

The purpose of this paper is to present the conditions which have prevailed during the rise of the cane-plantation and the transformation of the sugar-cane into the Kingdom of Valencia during the XVth century. The analysis is based on a rich material extracted from the royal archives and notarial documents concerning companies to exploit sugar-mill, agreements between peasants and landlords etc. The paper reveals the crucial role of the local muslim community in the culture of sugar-cane. Emphasis is put

\footnotetext{
${ }^{42}$ ARV Bailia 1165, fol. 406v. $407 \mathrm{r}$.
} 
on the fact that the competition with the cane-sugar producted in the Atlantic islands has brought the definitive decline of the cycle of the cane-plantation in Valencia on a large scale. On discussing the technological problems and relations of production, references are made on the main questions developped by the historiography about cane-sugar production in the Mediterranean area during the Middle Ages. 